\title{
Dynamic changes of T-lymphocyte subsets and the correlations with 89 patients with coronavirus disease 2019 (COVID-19)
}

\author{
Mingxiang Huang", Yao Wang", Jing Ye, Hongqiang Da, Sufang Fang, Lizhou Chen \\ Fuzhou Pulmonary Hospital \& Fujian Medical University Clinical Teaching Hospital, Fuzhou, China \\ Contributions: (I) Conception and design: M Huang; (II) Administrative support: L Chen; (III) Provision of study materials or patients: All authors; \\ (IV) Collection and assembly of data: All authors; (V) Data analysis and interpretation: Yao Wang; (VI) Manuscript writing: All authors; (VII) Final \\ approval of manuscript: All authors. \\ \#These authors contributed equally to this work. \\ Correspondence to: Lizhou Chen. Fuzhou Pulmonary Hospital \& Fujian Medical University Clinical Teaching Hospital, Fuzhou, China. \\ Email: hmg119@163.com.
}

Background: In December 2019, an outbreak of coronavirus disease 2019 (COVID-19), caused by a novel severe acute respiratory syndrome coronavirus 2 (SARS-CoV-2), occurred in Wuhan City, Hubei Province, China. The coronavirus has spread throughout the world, posing a severe threat to human health. By using flow cytometry, here we observed the dynamic changes of peripheral blood $\mathrm{T}$ lymphocyte subsets in COVID-19 patients, with an attempt to explore their roles in the pathogenesis of COVID-19 and their impacts on prognosis.

Methods: Eighty-nine COVID-19 patients were divided into a moderate group $(\mathrm{n}=70)$ and the severe/ critical group ( $\mathrm{n}=19)$ according to the disease severity. Furthermore, the severe/critical patients were divided into the improved group $(n=14)$ and unimproved group $(n=5)$ according to the outcomes. The absolute peripheral blood lymphocytes counts and subsets, including CD45+, CD3+, CD4+, and CD8+, in the acute phase, and flow cytometry measured the recovery phase for all patients. Then, the results were compared with those in the normal control group.

Results: The absolute counts of lymphocytes, T lymphocytes, and their subsets decreased during the acute phase in COVID-19 patients, especially in the severe/critical group. The T-lymphocyte count reached the lowest point on the 14th day in the severe/critical group. It rose with fluctuations to the normal level in the improved group as the immune function recovered; in the unimproved group, however, the T-lymphocyte count remained at a low level or even continued to decrease. The percentages of CD4+ and CD8+ T lymphocytes showed no visible change in the improved group; however, the percentage of CD8+ T cells dropped in the unimproved group, resulting in higher CD4+/CD8+ ratio.

Conclusions: $\mathrm{T}$ lymphocytes count, and their subsets can be used for monitoring the immune functions and predicting the prognosis of COVID-19 patients.

Keywords: Coronavirus disease 2019 (COVID-19); CD3; CD4; CD8

Submitted Jun 24, 2020. Accepted for publication Aug 27, 2020.

doi: $10.21037 / \mathrm{atm}-20-5479$

View this article at: http://dx.doi.org/10.21037/atm-20-5479

\section{Introduction}

In December 2019, patients with unexplained pneumonia were found in Wuhan City, Hubei Province, China. On January 7, 2020, the causative pathogen was identified as a virus subsequently named severe acute respiratory syndrome coronavirus 2 (SARS-CoV-2). On February 11, the World Health Organization officially named the disease caused by the novel coronavirus as coronavirus disease 2019 (COVID-19). On March 11, 2020, the WHO declared the COVID-19 outbreak a global pandemic (1-3). Currently, 
COVID-19 has spread to 211 countries and territories, with over 3.6 million confirmed cases and 240,000 deaths. The new cases and death tolls are still rising daily (4).

Coronaviruses are enveloped, positive-sense, singlestranded RNA viruses of $\sim 30 \mathrm{~kb}$. They infect a wide variety of host species. They are largely divided into four genera: $\alpha, \beta, \gamma$, and $\delta$. SARS-CoV-2 are classified to $\beta$ coronaviruses based on its genomic structure. Coronaviruses consist of four structural proteins; Spike (S), membrane (M), envelop (E) and nucleocapsid (N) (5). SARS-CoV-2 has $79 \%$ of the sequence homology to severe acute respiratory syndrome coronavirus (SARS-CoV) and $50 \%$ of the sequence homology to Middle East respiratory syndrome coronavirus (MERS-CoV). The fatality rate of SARS-CoV-2 (2.08\%) is lower than MERS-CoV (34.77\%) and SARS-CoV (10.87\%). Unlike SARS-CoV and MERS-CoV, which spreads in hospitals, SARS-CoV-2 is much more widely transmitted in the community and is more contagious (6-8). Early in infection, SARS-CoV-2 targets cells, such as nasal and bronchial epithelial cells and pneumocytes, through the viral structural spike (S) protein that binds to the angiotensin-converting enzyme 2 (ACE2) receptor. The type 2 transmembrane serine protease (TMPRSS2), present in the host cell, promotes viral uptake by cleaving ACE2 and activating the SARS-CoV-2 S protein, which mediates coronavirus entry into host cells. ACE2 and TMPRSS2 are expressed in host target cells, particularly alveolar epithelial type II cells. In later stages of infection, when viral replication accelerates SARS-CoV-2infectspulmonary capillary endothelial cells, accentuating endothelial barrier disruption, dysfunctional alveolar-capillary oxygen transmission, and impaired oxygen diffusion capacity. In severe COVID-19, fulminant activation of coagulation and consumption of clotting factors occur. In flamed lung tissues and pulmonary endothelial cells may result in microthrombi formation and contribute to the high incidence of thrombotic complications. The spectrum of COVID-19 varies from asymptomatic to clinical conditions including respiratory and gastrointestinal problems (9). The main manifestations include fever, cough, shortness of breath, muscle pain, drowsiness, headache, diarrhea, and nausea/vomiting. In severe cases, patients can develop acute respiratory distress syndrome and die of multiple organ failure (10-12).

It is known that the number of peripheral blood leukocytes is normal or decreases in COVID-19 patients, and the lymphocyte count declines. Among them, the
$\mathrm{T}$ lymphocytes, which are necessary for cell-mediated immunity, play a significant antiviral role. According to some previous studies, CD4+T cells, CD8+T cells, B cells, NK cells, and total lymphocytes were significantly reduced in patients with severe/critical type patients as compared to patients with mild/moderate type. The absolutely counts of CD4+ and CD8+ T cells both can independently predict prognosis of COVID-19 patients. Here, we detected and dynamically observed peripheral blood $\mathrm{T}$ lymphocyte subsets in 89 patients diagnosed with COVID-19, with an attempt to understand the changes of peripheral blood $\mathrm{T}$ lymphocyte subsets in COVID-19 patients and its significance. The results of the analysis can be used to intervene as early as possible in severe/critical patients to delay disease progression and improve the cure rate of severe/critical patients.

We present the following article in accordance with the MDAR reporting checklist (available at http://dx.doi. org/10.21037/atm-20-5479).

\section{Methods}

\section{Grouping}

Eighty-nine patients with clinically confirmed COVID-19, who were admitted to our hospital from January 23 to April 3, 2020, were enrolled in this study. Among them, there were 42 men and 47 women, aged 19-93 years [with a mean age of $(41.85 \pm 15.01)$ years]. The diagnosis was from the Diagnosis and Treatment Protocol for COVID-19 Infection (Trial Fifth Edition) released by the National Health Commission of China (13). The disease was moderate in 70 cases and severe/critical in 19 cases (including one death). After admission, all cases were continuously tested for T lymphocyte subsets (absolute count). According to the improvement of the disease during the recovery period, patients in the severe group were divided into improved severe/critical group $(\mathrm{n}=14)$ and unimproved severe/ critical group $(n=5)$. The control group consisted of 50 staff members [including 28 males and 22 females, aged (38t 13) years] of our hospital who took part in the health checkups during the same period. The study was conducted in accordance with the Declaration of Helsinki (as revised in 2013). The study was approved by the Ethical Committee of the Fuzhou Municipal Center for Disease Control (CDC), Fujian Province, China \{Approval No. 2020-001 [KY-01]\} and informed consent was taken from all the patients. 


\section{Typing of COVID-19 (Trial Fifth edition)}

The severity of COVID-19 was divided into the following types according to clinical symptoms, presence, and severity of pneumonia, respiratory failure, shock, and other organ failures:

(I) mild type (with slight clinical symptoms, and there is no appearance of pneumonia on imaging);

(II) moderate type (with fever, respiratory symptoms, and/or other conditions, and appearance of pneumonia is found on imaging);

(III) severe/critical type (i) $\mathrm{RR} \geq 30$ times/min; oxygen saturation $\leq 93 \%$ in resting-state; arterial partial pressure of oxygen $\left(\mathrm{PaO}_{2}\right)$ /concentration of oxygen $\left(\mathrm{FiO}_{2}\right) \leq 300 \mathrm{mmHg}$; (ii) respiratory failure requiring mechanical ventilation, shock, and other organ failure resulting in the treatment in the intensive care unit).

\section{Typing of improved severe/critical group and unimproved severe/critical group patients}

The inclusion criteria of the improved severe/critical group were as follows: (I) the temperature returned to normal for more than 3 days; (II) respiratory symptoms improved significantly; (III) lung imaging showed that inflammation was absorbed obviously.

The inclusion criteria of the unimproved severe/critical group were as follows: (I) death before discharge; (II) being in hospital because of severe bacterial pneumonia; (III) an increase in pulmonary parenchymal density, lung involvement gradually increased to consolidation, thickened pulmonary interstitial structures; (IV) using a ventilator to assist breathing.

\section{Measurements}

\section{Reagents and instruments}

A flow cytometer (FACSCalibur; BD, USA) was used. The reagents used included four-color fluorescent monoclonal antibody and FACS Erythrocyte Lysing Solution. BD Trucount ${ }^{\mathrm{TM}}$ tubes were used for determining absolute counts of leukocytes in blood.

\section{Experimental methods}

Heparin sodium-anticoagulated peripheral blood sample $(2 \mathrm{~mL})$ was collected for laboratory tests on the same day. If the sample could not be detected on the same day, it was stored at room temperature $\left(20-25^{\circ} \mathrm{C}\right)$ and tested within 48 hours.

For each specimen, $20 \mu \mathrm{L}$ of four-color fluorescentlylabeled monoclonal antibodies were added into the sample detection tube, followed by the addition of $50 \mu \mathrm{L}$ of wellmixed anticoagulated blood. The mixture was vortexed for $3 \mathrm{~s}$ and incubated at room temperature in the dark for 15 minutes. Then, red blood cell lysis solution $(10 \times)$ was added, and the mixture was incubated at room temperature in the dark for 15 minutes before flow cytometry detected it. The Multiset automatic analysis software (BD, USA) was used to detect $\mathrm{T}$ lymphocyte subsets in the specimen, and the percentage and absolute count of total $\mathrm{T}$ lymphocytes (CD3), the percentage and absolute count of Th cells $(\mathrm{CD} 3+\mathrm{CD} 4+\mathrm{CD} 8-)$, the percentage and absolute count of Ts cells (CD3+CD4-CD8+), and the total number of lymphocytes (CD45+) were reported, respectively. The dynamic changes of peripheral blood $\mathrm{T}$ lymphocyte subsets were compared between patients with moderate COVID-19 and those with severe/critical COVID-19. The dynamic changes of $\mathrm{T}$ lymphocytes in patients with moderate or severe/critical COVID-19 were observed from at admission to two months after discharge.

\section{Statistical analysis}

Statistical analysis was performed by using the SPSS 26.0 for Windows. Analysis of variance was performed to identify a significant difference between two different groups. A $\mathrm{P}$ value of less than 0.05 was regarded as statistically significant. Data are presented as mean \pm standard deviation.

\section{Results}

Comparison of age and gender between the moderate group and severe/critical group

Patients in the severe/critical group aged 35-88 years $[(63.3 \pm 15.32)$ years $]$, and patients in the moderate group aged 19-93 years $[(41.85 \pm 15.01)$ years] $(\mathrm{P}<0.05)$.

There were 11 males and 9 females in the severe/critical group and 31 males and 38 females in the moderate group $(\mathrm{P}>0.05)$.

\section{The number of mean days required for patients' nucleic acid tests turn from positive to negative}

Moderate group: (9.26 \pm 6.58$)$ days, improved severe/critical 
Table 1 Peripheral blood T lymphocytes at disease onset among patients with different COVID-19 types

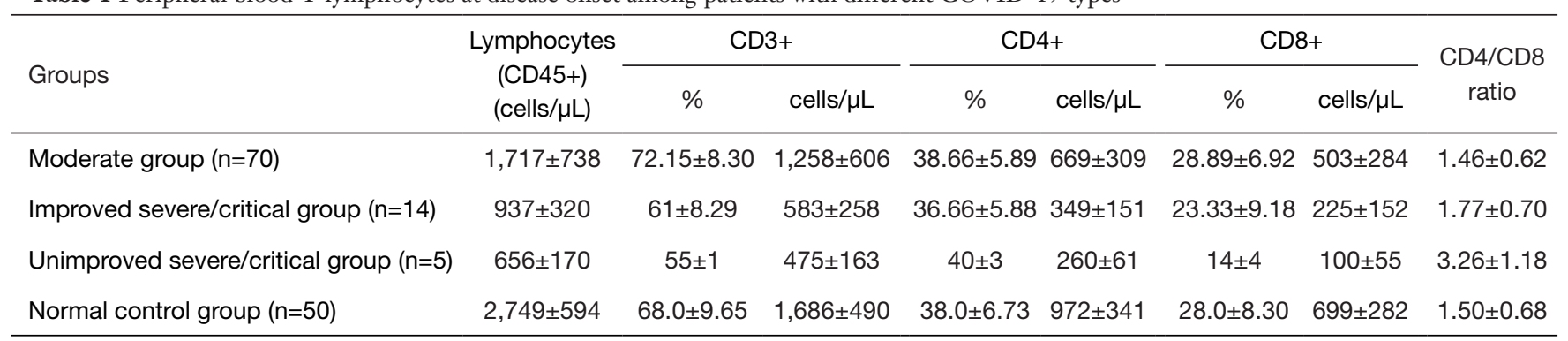

COVID-19, coronavirus disease 2019.

group: (13.78 \pm 2.65$)$ days, unimproved severe/critical group: $(21.00 \pm 2.91)$ days.

Absolutely, the mean days of unimproved severe/ critical group are almost twice as much as the mean days of moderate group $(\mathrm{P}<0.001$, the difference was statistically significant). Furthermore, the mean days of unimproved severe/critical group are much more than the mean days of improved severe/critical group $(\mathrm{P}<0.001$, the difference was statistically significant).

\section{The differences of CT images in three type patients}

CT images shows: ground glass opacities (GGOs) with rounded, multifocal, bilateral, peripheral distribution seem to be the most specific pattern found with COVID-19 pneumonia. In severe/critical patients, the frequency of bilateral lung involvement was higher, air bronchogram and multifocal involvement were more common. After clinical treatment, ground-glass opacities and airspace consolidation mostly disappeared, but in unimproved severe/critical groups, bilateral lung involvement with multifocal lesions, and more severe radiologic findings could be witnessed.

\section{Peripheral blood T lymphocytes at disease onset among patients with different COVID-19 types}

Significant $(\mathrm{P}<0.05)$ (Table 1).

\section{Dynamic changes of peripheral blood T-lymphocyte count in 89 COVID-19 patients}

T-lymphocytes count in the peripheral blood of COVID-19 patients decreased in the early stages of the disease. It reached the lowest on the 7 th day in the moderate group on the 14th day in the severe/critical group. Subsequently, the T-lymphocyte count gradually rose to the normal level in both moderate groups and improved severe/critical group; in the in unimproved severe/critical group, however, it remained low $(\mathrm{CD} 3+\mathrm{T}$ lymphocytes $<500 / \mu \mathrm{L})$ and continued to decline as the disease condition deteriorated (Figure 1).

\section{Absolute counts of peripheral blood T-lymphocytes and their subsets in severe/critical COVID-19 patients}

The mean values of both CD4+ and CD8+ T lymphocytes decreased in the severe/critical groups within 14 days after disease onset, especially in the unimproved severe/critical group (only $50 \%$ of those in the improved group; $\mathrm{P}<0.05$ ). Fourteen days after disease onset, the mean values of both CD4+ and CD8+ T lymphocytes increased in the improved group, but the difference was not statistically significant (both $\mathrm{P}>0.05$ ); the mean value of $\mathrm{CD} 4+\mathrm{T}$-cells remained low, and the mean value of CD4+ T-cells continued to decline in the unimproved group, although the differences were not statistically significant (both $\mathrm{P}>0.05$ ). The CD4/ CD8 ratios within 14 days after disease onset and after 14 days were not significantly different in the improved group $(\mathrm{P}>0.05)$ but showed a significant difference in the unimproved group $(\mathrm{P}<0.01)$ (Table 2$)$.

\section{Discussion}

A new type of coronavirus was isolated by Chinese authorities on January 7, 2020, and named "SARS-CoV-2" by the International Committee on Taxonomy of Viruses on February 11, 2020. As revealed by sequence analysis, SARS-CoV-2 is a positive-sense single-stranded RNA virus that belongs to the genus Betacoronavirus. It shares a $95 \%$ identity with a bat coronavirus (14). As a zoonotic pathogen, SARS-CoV-2 has 79.0\% nucleotide identity to SARS-CoV and $51.8 \%$ identity to MERS-CoV (8). Hoffmann et al. (15) 


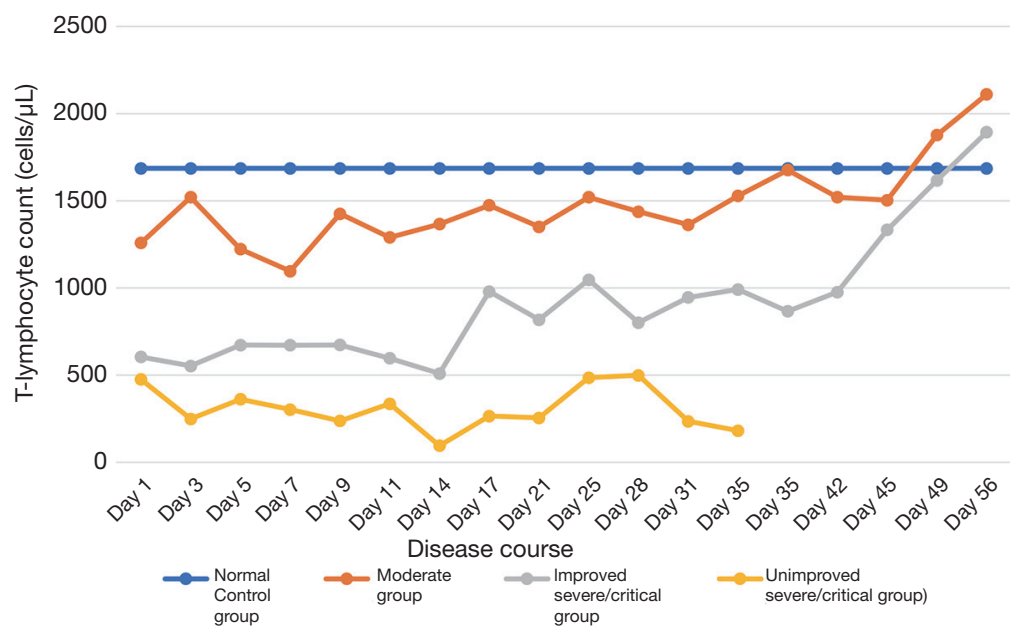

Figure 1 Dynamic changes of peripheral blood T-lymphocyte count in 89 COVID-19 patients. COVID-19, coronavirus disease 2019.

Table 2 Comparison of the absolute counts of peripheral blood T-lymphocytes and their subsets in severe/critical COVID-19 patients

\begin{tabular}{|c|c|c|c|c|c|c|c|c|}
\hline Groups & \multicolumn{2}{|c|}{ CD3+ (cells $/ \mu \mathrm{L})$} & \multicolumn{2}{|c|}{ CD4+ (cells $/ \mu \mathrm{L})$} & \multicolumn{2}{|c|}{ CD8+ (cells $/ \mu \mathrm{L})$} & \multicolumn{2}{|c|}{ CD4/CD8 ratio } \\
\hline $\begin{array}{l}\text { Within } 14 \text { days } \\
\text { after disease } \\
\text { onset }\end{array}$ & $634 \pm 326$ & $361 \pm 113$ & $350 \pm 180$ & $185 \pm 25$ & $250 \pm 161$ & $48 \pm 23$ & $1.80 \pm 0.82$ & $3.53 \pm 0.70$ \\
\hline $\begin{array}{l}\text { Normal control } \\
\text { group }\end{array}$ & \multicolumn{2}{|c|}{$1,686 \pm 490$} & \multicolumn{2}{|c|}{$972 \pm 341$} & \multicolumn{2}{|c|}{$699 \pm 282$} & \multicolumn{2}{|c|}{$1.50 \pm 0.68$} \\
\hline
\end{tabular}

COVID-19, coronavirus disease 2019.

have preliminarily elucidated the pathogenic mechanism of COVID-19: the protease TMPRSS2 activates the spike protein of SARS-CoV-2 before it binds to ACE2, which allows the virus to enter and infect lung cells. In our current study, we compared the age of disease onset between severe/critical and moderate COVID-19 patients. The age of patients in the severe/critical groups was older than that in the moderate group, which may be explained by the following fact: the T-lymphocyte count is negatively correlated with age, and thus the body's immune function decreases with age (16); accordingly, COVID-19 tends to affect older people more than younger ones.

The immune system supplies defense against infections via various immune cells, and lymphocytes are the key cells involved in the immune response. According to the different surface markers of lymphocytes, lymphocytes are be divided into $\mathrm{T}$ lymphocytes (CD3+), B lymphocytes (CD19+), and NK cells (CD56+). Cellular immunity, in particular those mediated by $\mathrm{T}$ lymphocytes, plays a key role in the fight against infections. According to their markers, $\mathrm{T}$ lymphocytes (CD3+) are further divided into $\mathrm{T}$ helper (Th) cells $(\mathrm{CD} 3+\mathrm{CD} 4+)$ and cytotoxic $\mathrm{T}(\mathrm{Tc})$ cells $(\mathrm{CD} 3+\mathrm{CD} 8+)(17)$. Many studies have shown that in patients with SARS (18), MERS (19), or COVID-19 (20), the immune system is attacked to varying degrees, and the immune system becomes disordered. In addition to humoral immunity, $\mathrm{T}$ cells are also important during viral infection. In the acute phase, virus clearance depends on specific CD4+ and CD8+ T lymphocytes, and the speed of virus clearance also depends on the response of $\mathrm{CD} 4+$ and CD8+ T lymphocytes (21). In the recovery phase, cellular immunity becomes more active, and humoral immunity no 
longer plays a dominant role; instead, $\mathrm{T}$ lymphocytes are activated to kill the viruses (22).

Both innate and adaptive immune responses participate in the defense against viruses. Initially, $\mathrm{T}$ cells bind to virus-specific antigen-presenting cells (APCs), which can last for several days after stable binding. Then, the $\mathrm{T}$ cells differentiate into effector $\mathrm{T}$ cells including $\mathrm{CD} 8+\mathrm{T}$ lymphocytes, which mainly kill host cells within which the pathogens [e.g., viruses] live and the CD4+ Th1 subgroup. The subgroup mainly mediates cellular immune response, during which the macrophages are activated to resist intracellular pathogens [e.g., viruses] jointly $(23,24)$. In most COVID-19 patients (especially those with severe/ critical conditions), a significant decrease in lymphocytes shows immune impairment. The decrease in T lymphocytes reflects the degree of immune imbalance, and a smaller T-lymphocyte count means weaker defense against SARSCoV-2 (25-27). Our current study has the same finding. Compared with the normal control group, the counts of $\mathrm{T}$ lymphocyte subsets were much lower in COVID-19 patients with different severities than in the normal control group, and the decline was prominent in the severe/critical groups. The T-lymphocyte count was as low as 273 cells $/ \mu \mathrm{L}$ in one patient, in whom severe lung infection and even multiple organ failure occurred after the COVID-19 was cured. The percentage of CD8+ T cells significantly dropped in the unimproved severe/critical group $(\mathrm{P}<0.05$, compared with the normal control group).

However, the percentages of other $\mathrm{T}$ lymphocyte subsets showed no such significant difference $(\mathrm{P}<0.05)$, suggesting the decrease in the CD8+ T-lymphocyte count was more significant than CD4+ T-lymphocyte count. Therefore, COVID-19 patients with lower total lymphocyte count and $\mathrm{T}$ lymphocyte subset counts tend to have more severe disease conditions. The decline of CD8+ T-lymphocyte counts is positively correlated with the reduced outcomes of COVID-19 patients.

$\mathrm{T}$ lymphocytes are essential for clearing the virus in patients with primary SARS-CoV infection; in particular, the virus-specific $\mathrm{T}$ lymphocytes play a crucial role in clearing the virus and alleviating clinical symptoms $(28,29)$. In our current study, we followed up the dynamic changes of $\mathrm{T}$ lymphocyte subset counts at different time points in 89 COVID-19 patients within two months after disease onset, which, to our knowledge, was the most extended observation in similar studies. Compared with the normal control group, the COVID-19 patients had lower T cell counts at disease onset, especially in the severe/critical groups (only $1 / 3$ of $\mathrm{T}$ cell count in the normal control group). In the moderate group, the T-lymphocyte count fell to the lowest point on the $7^{\text {th }}$ day and then rose with fluctuations, reaching the level in the normal control group on the $38^{\text {th }}$ day. In the severe/critical group, the T-lymphocyte count fell to the lowest point on the $14^{\text {th }}$ day; the average T-lymphocyte count was only 248 cells/ $\mu \mathrm{L}$ in the unimproved severe/critical group, and it was only 173 cells $/ \mu \mathrm{L}$ in one patient, who died of systemic multiple organ failure due to ARDS. In the improved severe/ critical group, the total T-lymphocyte count increased slowly after 14 days and showed a linear upward trend after 42 days, returning to a normal level on the $49^{\text {th }}$ day. The change in T-lymphocyte count showed opposite trends after the $28^{\text {th }}$ day: the improved group still showed an upward trend, whereas the unimproved group showed a sharp downward trend, which matched the deteriorating disease conditions. Thus, T-lymphocyte counts in the cured COVID-19 patients showed a reversible change, which first decreased and then gradually increased. These results reflect the process in which cellular immunity in the body was firstly destroyed or suppressed and then gradually returned normal. The T lymphocyte subsets in unimproved severe/critical patients remained low $(<500$ cells $/ \mu \mathrm{L})$, and the damaged cellular immune failed to recover, which was predictive of a poor prognosis. It has been reported that although the $\mathrm{T}$ lymphocytes were reduced in cured COVID-19 patients, the damage or suppression of the immune system was reversible, and the prognoses were good (30-32). The T-lymphocyte count was always below 500 cells $/ \mu \mathrm{L}$ in the unimproved group, which may be due to the following reasons: (I) the cells could not be normally activated when they were stimulated by antigen; (II) the cells were in an unresponsive state; or (III) many $\mathrm{T}$ lymphocytes died while the viruses were cleared. As the cellular immunity declined, the viruses in the body could not be adequately cleared, and a secondary infection could be easily induced, which eventually led to multiple organ failure and poor prognosis $(29,33)$.

Patients in the moderate group had a good prognosis after the recovery of the immune responses; however, the prognosis in the severe/critical groups could be either good or poor. As shown in Figure 1, the T-lymphocyte count reached the lowest point on the $14^{\text {th }}$ day in the severe/ critical groups. After 14 days, it rose with fluctuations in the improved group but remained low and finally dropped in the unimproved group, suggesting the $14^{\text {th }}$ day after disease onset might be a turning point in the disease course. 
Comparisons of the T lymphocyte subset count and CD4/ CD8 ratio showed that the mean $\mathrm{CD} 3+, \mathrm{CD} 4+$, and CD8+ T-lymphocyte counts significantly declined within the first 14 days after disease onset in the severe/critical groups, especially in the unimproved groups $(\mathrm{P}<0.05)$. Furthermore, the differences between improved and unimproved groups were significant $(\mathrm{P}<0.05)$, indicating that the cellular immunity was impaired in the early stages of the disease in the unimproved group. However, the CD4/CD8 ratio showed no significant differences between 14 days ago and 14 days later in the improve groups. During acute viral infections, virally derived peptides activate both naive CD8+ and CD4+ T cell proliferation and differentiation. As we know, effective viral clearance needs both CD8 effector $\mathrm{T}$ cell-mediated effective killing of virally infected cells and CD4 T cell-dependent enhancement of CD8 T and B cell responses. Following viral clearance, the majority of virusspecific $\mathrm{T}$ cells undergo apoptosis, then the number of $\mathrm{T}$ lymphocytes recovered slowly, but the dynamic changes of lymphocytes function in this process need further study (33). After 14 days, the mean CD3+, CD4+, and CD8+ T-lymphocyte count increased in the improved group, indicating that the cellular immunity was restored and the prognosis was good; in the unimproved group, the CD4+ T-lymphocyte count slightly increased, CD3+ and CD8+ T-lymphocyte counts did not rise but decreased, and the CD4/CD8 ratio continued to increase $(\mathrm{P}<0.001$, compared with the improved group).

In the later phases, CD8+ $\mathrm{T}$ cell count was below 150 cells $/ \mu \mathrm{L}$ in all the severely/critically ill patients who developed a secondary infection or multiple organ failure, and it might be less than 50 cells $/ \mu \mathrm{L}$ during the last test for the dead patient. Research has shown that SARS$\mathrm{CoV}-2$ is a positive-sense, single-stranded RNA virus. Its genome encodes four membrane proteins, of which the $\mathrm{S}$ protein is essential for the virus to invade the host cells. Angiotensin-converting enzyme (ACE) 2 exists in nonimmune cells (e.g., alveolar cells), and SARS-CoV-2 uses ACE2 for entry and the serine protease TMPRSS2 for S protein priming, thus causing damage to host cells (14). Most cytotoxic T lymphocytes (CTLs) are CD8+ T cells, which kill intracellular pathogens (e.g., viruses). CD8+ T cells specifically kill target cells by releasing toxic particles and death receptor pathways. Also, they can protect the body from secondary infections (34). It has also been found that $\mathrm{CD} 8+\mathrm{T}$ cells can be used as independent factors for predicting the severity and outcomes of COVID-19 in severe/critical cases (35). Similarly, we found that the prognosis was inferior in patients whose total T-lymphocyte count continued to be $<500$ cells $/ \mu \mathrm{L}$ and $\mathrm{CD} 8+$ T-lymphocyte count $<200$ cells $/ \mu \mathrm{L}$.

\section{Acknowledgments}

Funding: Supported by Fujian Provincial Science and Technology Program (2020Y0007) and Fuzhou Municipal Science and Technology Program (2020-XG-021).

\section{Footnote}

Reporting Checklist: The authors have completed the MDAR reporting checklist. Available at http://dx.doi.org/10.21037/ atm-20-5479

Data Sharing Statement: Available at http://dx.doi. org/10.21037/atm-20-5479

Conflicts of Interest: All authors have completed the ICMJE uniform disclosure form (available at http://dx.doi. org/10.21037/atm-20-5479). The authors have no conflicts of interest to declare.

Ethical Statement: The authors are accountable for all aspects of the work in ensuring that questions related to the accuracy or integrity of any part of the work are appropriately investigated and resolved. The study was conducted in accordance with the Declaration of Helsinki (as revised in 2013) and was approved by the Ethical Committee of the Fuzhou Municipal Center for Disease Control (CDC), Fujian Province, China Approval No. 2020-001 [KY-01]\} and informed consent was taken from all the patients.

Open Access Statement: This is an Open Access article distributed in accordance with the Creative Commons Attribution-NonCommercial-NoDerivs 4.0 International License (CC BY-NC-ND 4.0), which permits the noncommercial replication and distribution of the article with the strict proviso that no changes or edits are made and the original work is properly cited (including links to both the formal publication through the relevant DOI and the license). See: https://creativecommons.org/licenses/by-nc-nd/4.0/.

\section{References}

1. Lu H, Stratton CW, Tang YW. Outbreak of pneumonia of 
unknown etiology in Wuhan, China:The mystery and the miracle. J Med Virol 2020;92:401-2.

2. Coronaviridae Study Group of the International Committee on Taxonomy of Viruses. The species Severe acute respiratory syndrome-related coronavirus: classifying 2019-nCoV and naming it SARS-CoV-2. Nat Microbiol 2020;5:536-44.

3. World Health Organization Director-General's Opening Remarks at the Media Briefing on COVID-19-11 March 2020. Available online: https:/www.who.int/dg/speeches/ detail/who-director-general-s-openingremarks-at-themedia-briefing-on-covid-19---11-march-2020 (accessed on 11 March 2020).

4. WHO. Coronavirus disease (COVID-19) Situation Report-107, 6 May 2020.

5. Yuki K, Fujiogi M, Koutsogiannaki S. COVID-19 pathophysiology: A review. Clin Immunol 2020;215:108427.

6. Petrosillo N, Viceconte G, Ergonul O, et al. COVID-19, SARS and MERS:are they closely related? Clin Microbiol Infect 2020;26:729-34.

7. Xu Z, Shi L, Wang Y, et al. Pathological findings of COVID-19 associated with acute respiratory distress syndrome. Lancet Respir Med 2020;8:420-22.

8. Meo SA, Alhowikan AM, Al-Khlaiwi T, et al. Novel coronavirus 2019-nCoV: prevalence, biological and clinical characteristics comparison with SARS-CoV and MERSCoV. Eur Rev Med Pharmacol Sci 2020;24:2012-9.

9. van de Veerdonk FL, Netea MG, van Deuren M, et al. Kallikrein-kinin blockade in patients with COVID-19 to prevent acute respiratory distress syndrome. Elife 2020;9:e57555.

10. Klok FA, Kruip MJHA, van der Meer NJM, et al. Incidence of thrombotic complications in critically ill ICU patients with COVID-19. Thromb Res 2020;191:145-7.

11. Wiersinga WJ, Rhodes A, Cheng AC, et al. Pathophysiology, Transmission, Diagnosis, and Treatment of Coronavirus Disease 2019 (COVID-19): A Review. JAMA 2020. [Epub ahead of print].

12. Chen N, Zhou M, Dong X, et al. Epidemiological and clinical characteristics of 99 cases of 2019 novel coronavirus pneumonia in Wuhan, China: a descriptive study. Lancet 2020;395:507-13.

13. National Health Commission of China. Diagnosis and Treatment Protocol for COVID-19 Infection (Trial Fifth Edition). Available online: http://www.nhc.gov.cn/yzygj/ s7653p/202002/3b09b894ac9b4204a79db5b8912d4440. shtml
14. Singhal T, A Review of Coronavirus Disease-2019 (COVID-19). Indian J Pediatr 2020;87:281-6.

15. Hoffmann M, Kleine-Weber H, Schroeder S, et al. SARSCoV-2 Cell Entry Depends on ACE2 and TMPRSS2 and Is Blocked by a Clinically Proven Protease Inhibitor. Cell 2020;181:271-80.e8.

16. Davies JS, Thompson HL, Pulko V, et al. Role of CellIntrinsic and Environmental Age-Related Changes in Altered Maintenance of Murine T Cells in Lymphoid Organs. J Gerontol A Biol Sci Med Sci 2018;73:1018-26.

17. Brummelman J, Pilipow K, Lugli E. The Single-Cell Phenotypic Identity of Human CD8 and CD4 T Cells. Int Rev Cell Mol Biol 2018;341:63-124.

18. Cui W, Fan Y, Wu W, et al. Expression of lymphocytes and lymphocyte subsets in patients with severe acute respiratory syndrome. Clin Infect Dis 2003;37:857-9.

19. Shin HS, Kim Y, Kim G. et al. Immune Responses to Middle East Respiratory Syndrome Coronavirus During the Acute and Convalescent Phases of Human Infection. Clin Infect Dis 2019;68:984-92.

20. Xu B, Fan CY, Wang AL, et al. Suppressed T cellmediated immunity in patients with COVID-19: A clinical retrospective study in Wuhan, China. J Infect 2020;81:e51-60.

21. Channappanavar R, Zhao J, Perlman S. T cell-mediated immune response to respiratory coronaviruses. Immunol Res 2014;59:118-28.

22. Zhao J, Zhao J, Perlman S. T cell responses are required for protection from clinical disease and for virus clearance in severe acute respiratory syndrome coronavirus-infected mice. J Virol 2010;84:9318-25.

23. Zinkernagel RM. Immunology taught by viruses. Science 1996;271:173-8.

24. Cronkite DA, Strutt TM. The Regulation of Inflammation by Innate and Adaptive Lymphocytes. J Immunol Res 2018;2018:1467538.

25. Wang D, Hu B, Hu C, et al. Clinical Characteristics of 138 Hospitalized Patients With 2019 Novel CoronavirusInfected Pneumonia in Wuhan, China. JAMA 2020;323:1061-9.

26. Cecere TE, Todd SM, Leroith T. Regulatory T cells in arterivirus and coronavirus infections: do they protect against disease or enhance it? Viruses 2012;4:833-46.

27. Janice Oh HL, Ken-En Gan S, Bertoletti A, et al. Understanding the $\mathrm{T}$ cell immune response in SARS coronavirus infection. Emerg Microbes Infect 2012;1:e23.

28. Zhao J, Zhao JX, Van Rooijen N, et al. Evasion by stealth: inefficient immune activation underlies poor $\mathrm{T}$ cell 
response and severe disease in SARS-CoV-infected mice. PLoS Pathog 2009;5:e1000636

29. Qin C, Zhou L, Hu Z, et al. Dysregulation of Immune Response in Patients With Coronavirus 2019 (COVID-19) in Wuhan, China. Clin Infect Dis 2020;71:762-8.

30. He Zh, Zhao C, Dong Q, et al. Effects of severe acute respiratory syndrome (SARS) coronavirus infection on peripheral blood lymphocytes and their subsets. Int J Infect DiS 2005;9:323-30.

31. Wang F, Hou H, L Yi, et al. The laboratory tests and host immunity of COVID-19 patients with different severity of illness. JCI Insight 2020;5:e137799.

32. Li T, Qiu Z, Han Y, et al. Rapid loss of both CD4+ and

Cite this article as: Huang M, Wang Y, Ye J, Da H, Fang S, Chen L. Dynamic changes of T-lymphocyte subsets and the correlations with 89 patients with coronavirus disease 2019 (COVID-19). Ann Transl Med 2020;8(18):1145. doi: 10.21037/ atm-20-5479
CD8+ T lymphocyte subsets during the acute phase of severe acute respiratory syndrome. Chin Med J (Engl) 2003;116:985-7.

33. Vardhana SA, Wolchok JD. The many faces of the anti-COVID immune response. J Exp Med 2020;217:e20200678.

34. Schmidt ME, Varga S M, The CD8 T Cell Response to Respiratory Virus Infections. Front Immunol 2018;9:678.

35. Wang F, Nie J, Wang H, et al. Characteristics of Peripheral Lymphocyte Subset Alteration in COVID-19 Pneumonia. J Infect Dis 2020;221:1762-69.

(English language editor: J. Chapnick) 